\title{
A confiança interorganizacional nas compras
}

\author{
The interorganizational trust in purchases
}

\author{
Marli de Fátima Ferraz da Silva Tacconi ${ }^{1,2}$ \\ Fernando Dias Lopes ${ }^{3}$ \\ Anderson Luiz Rezende Mól ${ }^{1}$ \\ Ernesto Alexandre Tacconi Neto ${ }^{4}$
}

\begin{abstract}
Resumo: O conceito de confiança interorganizacional apresenta lacunas que requerem maior número de pesquisas para que se possa construir uma teoria coerente que apoie as estratégias de cooperação e integração da cadeia de suprimentos. Esta pesquisa tem o intuito de explicar os antecedentes da confiança interorganizacional pela relação entre os construtos desempenho operacional, características organizacionais, compartilhamento de valores e relacionamento interpessoal nas compras realizadas pelas indústrias de transformação. No que se refere aos aspectos metodológicos, o estudo assume a forma de uma pesquisa descritiva, do tipo survey, e causal, de cunho teórico-empírico, com caráter explicativo, desenvolvida por meio da modelagem de equações estruturais. Os questionários foram aplicados entre os informantes-chave, utilizando-se uma amostragem aleatória simples desenvolvida nas indústrias de transformação (CNAE 10 a 33) localizadas na região da Grande Natal, que constituem um importante setor de atividade econômica com capacidade de gerar dinamismo na economia. Os primeiros resultados das análises demonstram o fenômeno da confiança interorganizacional, a qual consiste em empresas compradoras acreditarem na empresa fornecedora, sentirem-se seguras em relação a esta. $\mathrm{O}$ modelo inicial proposto não apresentou ajustamento, precisando ser reespecificado. Esse modelo reespecificado demonstra que os resultados suportam a explicação de que a confiança interorganizacional nas compras depende diretamente do relacionamento interpessoal, do compartilhamento de valores e do desempenho operacional. Conclui-se que essa confiança pode ser explicada por um conjunto de interações entre esses três determinantes, sendo o ponto central o relacionamento interpessoal, que apresentou o maior coeficiente de trajetória para o fator.
\end{abstract}

Palavras-chave: Confiança interorganizacional. Compras. Relações comprador-fornecedor. Equações estruturais. Logística. Gestão da cadeia de suprimentos.

\begin{abstract}
The concept of inter-organizational trust presents gaps that require further research so that a coherent theory to support the strategies of cooperation and integration of the supply chain can be built. In this sense, this research aims to explain the antecedents of interorganizational trust by the relationship between the constructs operational performance, organizational characteristics, sharing of values, and interpersonal relationships, in the purchases made by processing industries. With regard to methodological aspects, this study takes the form of a descriptive survey type, as well as a causal, theoretical and empirical research, with explanatory character, developed through structural equation modeling. The questionnaires were administered to key informants, using simple random sampling developed in processing industries (CNAE 10-33) located in the region of Natal, which is an important sector with capacity to generate economic dynamism. The first results of the analyses demonstrate the phenomenon of inter-organizational trust, in which buying companies trust their suppliers and feel safe about the process. The initial proposed model showed no adjustment, and it had to be re-specified. This re-specified model demonstrates that the results support the explanation that trust in interorganizational purchases depends directly on interpersonal relationships, sharing of values, and operational performance. We concluded that this trust can be explained by a set of interactions between these three determinants, with interpersonal relations as the central point, which presented the highest path coefficient for the factor.
\end{abstract}

Keywords: Interorganizational trust. Purchases. Buyer-supplier relationships. Structural equation. Logistics. Supply chain management.

\footnotetext{
${ }^{1}$ Programa de Pós-graduação em Administração - PPGA, Universidade Federal do Rio Grande do Norte - UFRN, Campus Universitário Lagoa Nova, Rod. BR 101, Km 1, Lagoa Nova, CP 1570, CEP 59072-970, Natal, RN, e-mail: mol@ufrnet.br

2 Instituto Federal de Educação, Ciência e Tecnologia do Rio Grande do Norte - IFRN, Campus Natal - Central, Av. Senador Salgado Filho, 1559, Tirol, CEP 59015-000, Natal, RN, Brasil, e-mail: marli.tacconi@ifrn.edu.br

${ }_{3}^{3}$ Programa de Pós-graduação em Administração, Universidade Federal do Rio Grande do Sul - UFRGS, Rua Washington Luiz, 855, Centro, CEP 90010-460, Porto Alegre, RS, e-mail: fdlopes@ea.ufrgs.br

${ }^{4}$ Instituto Federal de Educação, Ciência e Tecnologia do Rio Grande do Norte - IFRN, Campus Natal - Zona Note, Rua Brusque, 2926, Conjunto Santa Catarina, Potengi, CEP 59112-490, Natal, RN, Brasil, e-mail: ernesto.tacconi@ifrn.edu.br
}

Recebido em 5/5/2012 — Aceito em 5/6/2013

Suporte financeiro: Nenhum. 


\section{Introdução}

A forma de suprir a empresa foi gerando mudanças em antigos conceitos, o que resultou na inclusão de novas formas de gestão consideradas mais eficientes, como o relacionamento cooperativo com os fornecedores. Essa questão é exposta por Morrissey e Pittaway (2006), quando afirmam que as compras têm sido reconhecidas como um importante fator de gestão e que vêm se baseando em concepções de colaboração.

Um problema importante, na prática, é que, apesar de a colaboração com os fornecedores ser reconhecida como importante, as estratégias organizacionais, bem como a cultura, as políticas, os sistemas e os procedimentos das empresas, parecem reforçar o comportamento concorrencial. Criar uma capacidade de colaborar com os fornecedores requer mudanças na forma organizacional, e não simplesmente pequenas intervenções muito específicas nas relações interorganizacionais (KNIGHT, 2000).

As compras organizacionais, em uma visão estratégica, têm os relacionamentos cooperativos com os fornecedores como o ponto-chave para uma gestão eficaz na cadeia de suprimentos. Conforme Hernandez e Santos (2007), a confiança no parceiro, nas relações de troca, leva à manutenção de relacionamentos mais longos e estáveis, instiga a orientação de longo prazo, diminui a incerteza e o nível de conflitos, além de aumentar a satisfação com o relacionamento, o comprometimento e as intenções futuras de compra.

Chen, Paulraj e Lado (2004) destacam que a capacidade da confiança em gerar relações de cooperação a longo prazo e, simultâneamente, reduzir os custos de transação, aumentando o valor nas trocas, precisa ser explicitamente mais medida e avaliada como parte integrante do construto da gestão de suprimentos.

Para a gestão da cadeia de suprimentos, é necessário que as organizações trabalhem em conjunto, para encontrar maneiras de aumentar o valor apresentado aos clientes finais, no qual a presença da confiança tem o poder de melhorar substancialmente as oportunidades de um bom desempenho (BOWERSOX; CLOSS; COOPER, 2007).

No entanto, segundo Hill et al. (2009), os relacionamentos estão entre as áreas de maior carência para as empresas. Isso revela que, além dos problemas logísticos, tais como transporte, armazenagem e gestão de estoques, as questões referentes à ética, à confiança e ao contrato psicológico entre comprador e fornecedor são fundamentais.

$\mathrm{Na}$ exposição de um painel, Correa (2010) apresentou as oportunidades de pesquisa para a área de operações descrevendo a questão da gestão de riscos na rede como a grande oportunidade do momento, porque a globalização teria aumentado os riscos de ruptura nas cadeias de suprimento. Entre os riscos que mais preocupam as organizações, em primeiro lugar, está "Disponibilidade, custo e qualidade da mão de obra" (43\%), seguido por "Legislação e regulamentos" (36\%) e, em terceiro lugar, "Confiabilidade dos fornecedores" (33\%), em um ranking com doze alternativas.

Além disso, para o European Group for Organizational Studies (2011), uma questão relevante nessa área seria: "Quais são os antecedentes da confiança organizacional e institucional?". Porque ainda não existe um consenso claro sobre o conceito de confiança, ou digno de confiança, no nível organizacional, nem uma teoria coerente, um modelo consensual e/ou pesquisas empíricas suficientes para orientar um entendimento global sobre a confiança organizacional.

Essa lacuna, em relação à necessidade de melhor entendimento sobre a confiança entre as organizações, vem ao encontro desta pesquisa, que se propõe contribuir com uma proposição desse fenômeno no relacionamento comprador-fornecedor, a fim de dar suporte às estratégias que são desenvolvidas para integrar a cadeia de suprimentos na realidade empresarial.

Nessa direção, o objetivo da pesquisa consiste em explicar os antecedentes da confiança interorganizacional pela relação entre os construtos desempenho operacional, características organizacionais, compartilhamento de valores e relacionamento interpessoal nas compras realizadas pelas indústrias de transformação.

Como objetivos específicos, pretende-se: propor um modelo de confiança interorganizacional nas compras; identificar o poder preditivo das variáveis latentes desempenho operacional, características organizacionais, compartilhamento de valores e relacionamento interpessoal sobre a confiança interorganizacional nas compras; e verificar a aderência do modelo teórico às evidências empíricas nas indústrias de transformação da Grande Natal (RN).

\section{Compras organizacionais e confiança interorganizacional}

A gestão de compras e suprimentos tem assumido um papel fundamental na gestão estratégica da cadeia de suprimentos (BAIER; HARTMANN; MOSER, 2008; CHEN; PAULRAJ; LADO, 2004). Essa função faz parte do processo de logística, sendo responsável pela negociação com os fornecedores em questões como preço, prazo, material promocional, giro de estoque, entre outras que fornecem respaldo para um preço final do produto mais competitivo. Além disso, organiza as condições de pagamento, verifica os melhores fornecedores e, consequentemente, supre a organização de bens ou serviços com a qualidade e a quantidade correta para todo o processo em si. 
Nenhuma organização é capaz de gerar todos os recursos de que necessita e, dessa forma, não é autossuficiente, havendo a necessidade de desenvolver parceiras com outras firmas para complementar seus recursos. Para uma firma, os recursos incluem as instalações, os processos organizacionais, a informação, a marca, o conhecimento tecnológico da empresa, o maquinário, o capital financeiro, entre outros (BARNEY, 1991).

Segundo Arkader (2004), a compra, como atividade de natureza burocrática e pouco atraente à gerência, passou a ser revalorizada a partir das práticas justin-time das montadoras japonesas, que apresentaram uma nova visão sobre as questões de fornecimento ou suprimento, expandindo-a para os mais diferentes setores. Por isso, o conjunto de práticas realizadas de forma estratégica com a finalidade de suprir a empresa com os bens e serviços que são necessários pode ser chamado de "gerência de suprimentos".

Nessa direção, os processos de compras organizacionais - ou melhor, a gerência de suprimentos - são considerados atividade estratégica pelas empresas, em virtude de sua capacidade de reduzir os gastos totais e do desenvolvimento de relacionamentos entre compradores e vendedores, que possibilitam melhoria na cadeia de suprimentos.

A função compras, como outras áreas na logística, era desempenhada por especialistas que eram considerados eficientes à medida que alcançavam bons indicadores na redução dos custos de transporte, dos estoques e na aquisição de produtos ou serviços pelo menor preço. No entanto, com a elevação do nível de exigência dos clientes e com a abertura dos mercados, a logística precisa passar a dar respostas mais eficazes, o que tem gerado a necessidade de integração de todas as atividades de movimentação e armazenamento. Por isso mesmo, seu estudo não se limita a uma única função como marketing ou operações, e sim a uma área de integração desses distintos enfoques (FIGUEIREDO; ARKADER, 1998).

A intensificação na competição expôs defeitos do velho método de produção e da relação com os fornecedores, nos quais a integração vertical em indústrias como a automobilística levava à inflexibilidade. Perseguir como estratégia a minimização dos custos também reduzia a capacidade dessas montadoras de inovar. Diante desse panorama, as grandes empresas aumentaram o foco no setor de compras, especialmente por razões relacionadas à velocidade e à criatividade. A queda dos lucros e a perda de fatia de mercado fizeram com que as montadoras escolhessem sacrificar alguns de seus poderes de barganha perante os fornecedores para obterem mais inovações nos produtos (POWELL, 1987).

Os compradores educados nos conceitos de custo total de aquisição ou propriedade têm uma visão mais sofisticada das relações na cadeia de suprimentos, evitando o estreito foco no preço e procurando influenciar os negócios em termos de qualidade, a fim de evitar os custos associados com a não conformidade dos produtos ou serviços (MORRISSEY; PITTAWAY, 2006).

Os compradores organizacionais, nas compras rotineiras, relutam em mudar de fornecedores caso não haja uma razão justificável, o que tende a criar relacionamentos de longo prazo. Nesse sentido, os compradores modernos estão procurando fornecedores com os quais possam trabalhar em benefício mútuo. As grandes organizações de compras, cada vez mais, esperam contratar fornecedores-chave, selecionados após avaliação minuciosa e situados em locais próximos a suas instalações (BAILY et al., 2000).

Essa relutância pode ser explicada pela economia dos custos de transação, que difere das abordagens mais comportamentais da teoria da firma, porque utiliza uma combinação de abordagens econômicas e organizacionais para estudar as firmas e os mercados, suplantando a preocupação com o mercado como uma simetria em todas as formas de organização. Essa teoria descreve que as partes dos contratos comerciais refletem uma percepção sobre a natureza das relações existentes, incluindo a consciência do perigo potencial do contrato. No entanto, devido ao fato de os contratos complexos serem inevitavelmente incompletos, é impossível, ou proibitivamente caro, prever todas as contingências possíveis de forma antecipada e, dessa forma, muito da ação contratual é suportado posteriormente pelas estruturas de governança (WILLIAMSON, 1996).

Os relacionamentos na cadeia de suprimentos, que englobam os relacionamentos entre compradores e fornecedores, estão entre as áreas mais complexas e menos compreendidas das operações logísticas. Argumenta-se que "[...] os executivos devem aprender a gerenciar por meio da persuasão e da cooperação, em vez da coerção." (BOWERSOX; CLOSS, 2008, p. 109).

Assume-se, neste artigo também, que a confiança é um dos conceitos-chave na competição baseada no conhecimento, na era das redes (SEPPÄNEN; BLOMQVIST; SUNDQVIST, 2007), uma vez que as firmas que são capazes de moldar suas configurações de relacionamentos internos e externos não são facilmente imitadas ou substituídas. Esse aspecto, por sua vez, pode traduzir-se em vantagens competitivas sustentáveis (WALTER; LECHNER; KELLERMANNS, 2007), as quais decorrem de recursos organizacionais que não estão disponíveis para serem comprados no mercado (BARNEY, 1991).

Gerenciar os relacionamentos e coordenar as atividades por meio dos múltiplos participantes independentes é um grande desafio para qualquer gestor. Os documentos legais são incapazes de 
antecipar todas as possíveis ocorrências em todas as atividades cooperativas e, ainda assim, manter a intensa flexibilidade necessária para uma interação interorganizacional. Nesse sentido, nas relações entre comprador e fornecedor, a ênfase recai muito mais sobre a confiança do que sobre os contratos formais (HILL et al., 2009). Por isso, algum nível de confiança é necessário para que repetidas operações interorganizacionais aconteçam (LAAKSONEN; PAJUNEN; KULMALA, 2008).

A confiança decorre da disponibilidade de uma pessoa ou grupo para relacionar-se com outra na crença de que as ações dessa pessoa sejam mais benéficas do que prejudiciais, mesmo que isso não possa ser garantido (CHILD, 2001).

A confiança é uma expectativa 'positiva' e 'confiante' sobre a outra parte e uma 'disponibilidade a aceitar a vulnerabilidade' nas relações, em condições de interdependência e risco. (LEWICKI; TOMLINSON; GILLESPIE, 2006, p. 1014).

No entanto, ter confiança em alguém não significa cem por cento de confiança de que a pessoa fará qualquer tarefa e em qualquer circunstância, mesmo porque os indivíduos entendem que não há nada que esteja totalmente certo neste mundo (DAS; TENG, 2004).

Confiar em outras pessoas é uma decisão difícil. A confiança em indivíduos tem sido bem compreendida como sendo afetada pelas características destes, pelas relações interpessoais e pelo ambiente institucional, mas a confiança no contexto organizacional tende a ser mais complexa porque precisa considerar a confiabilidade dos membros organizacionais, que são moldados pelos processos, pela estrutura e pela cultura organizacional, implicando a necessidade de considerar os efeitos do contexto organizacional sobre o comportamento individual (PERRONE; ZAHEER; McEVILY, 2003).

Uma definição de confiança interorganizacional é

[...] a extensão com que os membros de uma organização retêm uma confiança coletiva orientada em direção a outra organização. (ZAHEER; HARRIS, 2006, p. 170).

Nesse alinhamento, para esta pesquisa, a confiança nas relações de compras entre empresas significa que os membros de uma organização (compradores e diretores) acreditam na empresa fornecedora, sentem-se seguros em relação a esta.

Viana e Baldi (2008) afirmam que, em uma cadeia de suprimentos, cujos relacionamentos se configuram nas relações cliente-fornecedor, é possível a ocorrência de diferentes tipos de laços interorganizacionais. Nas relações entre clientes e fornecedores, esses laços podem ocorrer na forma de laços arm's length, em exemplos como aquisição de material de expediente e serviços de manutenção. Contudo as relações mais importantes para o sucesso em uma cadeia de suprimentos seriam aquelas baseadas em laços imersos, que incluem os relacionamentos pessoais e as redes sociais entre os membros organizacionais.

Um resultado importante confirmado na pesquisa de Ganesan e Hess (1997) foi que os compradores organizacionais fazem uma distinção entre confiança no vendedor e confiança na organização fornecedora. Segundo eles, esse achado é relevante, porque valida a visão multidimensional da confiança.

Outro estudo, realizado por Tacconi, Lopes e Ramos (2009), mostrou que, na percepção do gestor de compras de uma grande empresa do setor de alimentos, a confiança interorganizacional está diluída igualmente entre a pessoa com quem ele se relaciona e a organização fornecedora. Contudo, quando a organização perde a confiança interpessoal, existe a possibilidade de contato com um novo indivíduo nessa mesma organização para realizar as negociações. Dessa forma, a confiança interpessoal e a interorganizacional são construtos distintos, mas que estão imbricados em um mesmo sistema de relações.

\section{Antecedentes da confiança interorganizacional nas compras}

Os estudos empíricos que investigaram a confiança com foco na relação comprador-fornecedor, ou relações de troca, foram levantados nos anos de 2009 e 2011 na página de busca do Web of Science. Esse levantamento identificou que as pesquisas quantitativas de Morgan e Hunt (1994), Doney e Cannon (1997), Zhao e Cavusgil (2006) e Nyaga, Whipple, Lynch (2010), bem como a pesquisa qualitativa de Tacconi et al. (2011), foram as que efetivamente investigaram os antecedentes da confiança interorganizacional. Aliados aos achados desses autores foram acrescidos outros indicadores considerados significativos para a formação e a definição das hipóteses.

O clássico trabalho de Morgan e Hunt (1994) testou várias hipóteses relacionadas aos fatores que influenciam a confiança e o comprometimento nas relações de troca e verificaram que a comunicação afeta positivamente esse fenômeno. Essa pesquisa utilizou como principal referência o trabalho de Doney e Cannon (1997), os quais concluíram que a confiança da firma compradora na fornecedora está relacionada à customização de produtos ou serviços. Quanto mais um fornecedor se disponibiliza a atender às exigências do comprador, maior tende a ser o nível de confiança. Nessa direção, a pesquisa de Tacconi et al. (2011) apresenta que um bom desempenho é fator gerador de confiança interorganizacional.

O compartilhamento de informações foi uma variável que se apresentou como significativa nos 
trabalhos de Laaksonen, Pajunen e Kulmala (2008) e Nyaga, Whipple, Lynch (2010). Já Zhao e Cavusgil (2006) apontam como significativas a orientação para o cliente, que inclui fornecer informações precisas, e a coordenação de todas as funções da empresa em prol do atendimento ao cliente. Quando um fornecedor é percebido com esse tipo de comportamento, ele tende a ser credível e benevolente e, dessa forma, é visto pelo fabricante como digno de confiança. Além dessas variáveis, Laaksonen, Pajunen e Kulmala (2008) apresentam a consistência nas entregas de produtos de forma oportuna e exata como uma das condições propícias para o surgimento da confiança.

Estas variáveis, como nível de comunicação, customização, consistência nas entregas, descritas como significativas para a geração da confiança, são aspectos considerados nesta pesquisa como desempenho operacional do fornecedor. Nesse sentido, propõe-se que:

Hipótese H1: Um bom desempenho operacional influencia positivamente como antecedente da confiança interorganizacional nas compras.

A pesquisa de Doney e Cannon (1997) relacionou a confiança ao tamanho da empresa: quanto maior o tamanho da organização fornecedora, maior tende a ser o nível de confiança do comprador. Em relação ao vendedor, a confiança é oriunda de maior expertise dele, que possibilita um bom desenvolvimento das relações. A colocação do cliente em primeiro lugar, em que o comprador se sente especial, aparece como fator positivo na geração de confiança nas pesquisas de Zhao e Cavusgil (2006) e Tacconi et al. (2011), enquanto Laaksonen, Pajunen e Kulmala (2008) indicam a boa reputação do fornecedor como uma das condições propícias para o surgimento da confiança.

Gulati e Singh (1998), avaliando a influência da confiança como um dos fatores que podem afetar a escolha da estrutura de governança das alianças, verificaram que a comparação entre alianças locais e de outras regiões foi amplamente consistente em relação à expectativa de que a confiança é maior em função da proximidade. Outra pesquisa, desenvolvida por Nyaga, Whipple e Lynch (2010), concluiu que o esforço no relacionamento conjunto, como o estabelecimento de metas de planejamento, é um dos pontos que levam à criação de confiança.

Tomados em conjunto, esses resultados sugerem que algumas características organizacionais da empresa fornecedora têm potencial para anteceder a confiança. Com base neles, apresenta-se a seguinte hipótese:

Hipótese H2: As características organizacionais do fornecedor influenciam positivamente como antecedente da confiança interorganizacional nas compras.

Morgan e Hunt (1994) verificaram que, quanto maior o compartilhamento de valores, maior será o nível de confiança nas relações de compra. E, de forma inversa, quanto maior o comportamento oportunístico, menor será a geração de confiança. Nessa mesma direção, no trabalho de Tacconi et al. (2011), a ética empresarial emergiu como um dos geradores, porque a percepção de que a organização fornecedora tem condutas morais adequadas eleva a credibilidade entre as partes e tende a reduzir os controles nas transações comerciais.

Outras variáveis destacadas no trabalho de Laaksonen, Pajunen e Kulmala (2008) foram os investimentos em ativos específicos e a justiça nos relacionamentos, com o compartilhamento dos riscos e acordos na distribuição dos lucros, como variáveis com potencial de propiciar o surgimento da confiança nas relações cliente-fornecedor. Da mesma forma, a pesquisa de Nyaga, Whipple, Lynch (2010) também apresenta os ativos específicos investidos pelo fornecedor para atender ao comprador como fator gerador de confiança. Além desses achados, Child e Möllering (2003) descobriram que a transferência de práticas empresariais aumenta a confiança.

Com base nesses argumentos, sugere-se a seguinte proposição:

Hipótese 3: O compartilhamento de valores influencia positivamente como antecedente da confiança interorganizacional nas compras.

Doney e Cannon (1997) buscaram fornecer novas ideias sobre como a confiança se desenvolve e como influencia o comportamento de compra industrial nos Estados Unidos e verificaram que essa confiança se origina de maior simpatia do vendedor, da frequência com que ele mantém contatos empresariais e da semelhança entre comprador e fornecedor. Já as repetidas interações entre compradores e fornecedores também foram destacadas por Laaksonen, Pajunen e Kulmala (2008) como geradoras da confiança.

Uzzi e Gillespie (2002) enfatizam que as transações de amizade e em redes sociais criam expectativas de confiança e de reciprocidade. Da mesma maneira, Child e Möllering (2003) também enfatizam que o desenvolvimento de um bom relacionamento pessoal tende a aumentar esse tipo de confiança. Nesse alinhamento, a pesquisa de Tacconi et al. (2011) apresenta a proximidade nos relacionamentos, nos quais desenvolver boas relações pessoais com os compradores organizacionais, a fim de estreitar esses relacionamentos, leva à geração de confiança interorganizacional. Portanto, a hipótese de pesquisa associada a essas variáveis se configura como:

Hipótese 4: O relacionamento interpessoal influencia positivamente como antecedente da confiança interorganizacional nas compras.

Essas pesquisas formam o principal aporte teórico para a explicação dos antecedentes da confiança nas relações comprador-fornecedor que originaram as quatro hipóteses centrais deste trabalho. 


\section{Procedimentos metodológicos}

A presente pesquisa é de cunho teórico-empírico e, do ponto de vista metodológico, pode ser classificada como descritiva, do tipo survey, e causal, porque se destina a descrever as variáveis que geram a confiança nas compras interorganizacionais, bem como medir o relacionamento entre essas variáveis.

A pesquisa se restringiu ao universo de 1.064 indústrias de transformação, de diversos portes, da cidade de Natal e do entorno da capital, que inclui as cidades de Parnamirim, Macaíba, São Gonçalo do Amarante e Extremoz, compreendendo os códigos CNAE de divisão 10 a 33, seção C, disponível na página eletrônica da Federação Nacional das Indústrias do Estado do Rio Grande do Norte (2011). O setor de atividade econômica industrial é importante por sua capacidade de gerar renda, investimento e desenvolvimento para uma localidade. Além disso, o número de fornecedores disponíveis para as indústrias tende a ser mais restrito e, portanto, o desenvolvimento de relações de longo prazo entre essas organizações e seus fornecedores tem um impacto maior nas estratégias empresariais. Finalmente, pode-se afirmar que as características da indústria de transformação do Rio Grande do Norte, em termos de tamanho, atividades envolvidas, nível de desenvolvimento tecnológico e tipo de propriedade, não apresenta discrepância significativa em relação às das indústrias dos principais estados do Nordeste, bem como de muitos outros estados da Federação. Assim, com exceção dos aspectos culturais, que exercem certa influência sobre as relações de confiança, as demais variáveis estão controladas e permitem certa generalização dos resultados para uma população mais ampla, embora isso não seja objetivo deste estudo.

Em relação ao processo de coleta dos dados, iniciou-se com o levantamento das indústrias, passando-se por várias etapas, conforme fluxo apresentado na Figura 1.

A participação na pesquisa era confirmada por contato telefônico, conforme a Figura 1. E, caso afirmativo, era agendado o dia e o horário mais convenientes para a aplicação dos questionários junto aos compradores e diretores. O levantamento totalizou 237 questionários válidos, compreendendo o período de 25 de julho a 23 de setembro de 2011, para as ligações, e até 30 de setembro de 2011 para a coleta.

O questionário compreendia 32 questões referentes às variáveis manifestas (Quadro 1) e uma sobre o nível de confiança interorganizacional, em escala de intensidade intervalar equivalente aos itens da escala de Likert, com valores rotulados de 1 (discordo totalmente) até 10 (concordo totalmente), subdivididas de meio em meio ponto, bem como questões de múltipla escolha e uma questão dicotômica, para as perguntas relacionadas ao perfil.

Quanto a sua natureza, esta investigação, de caráter explicativo, se desenvolveu por meio da modelagem de equações estruturais - SEM, com o recurso do software IBM SPSS Amos 18.0.

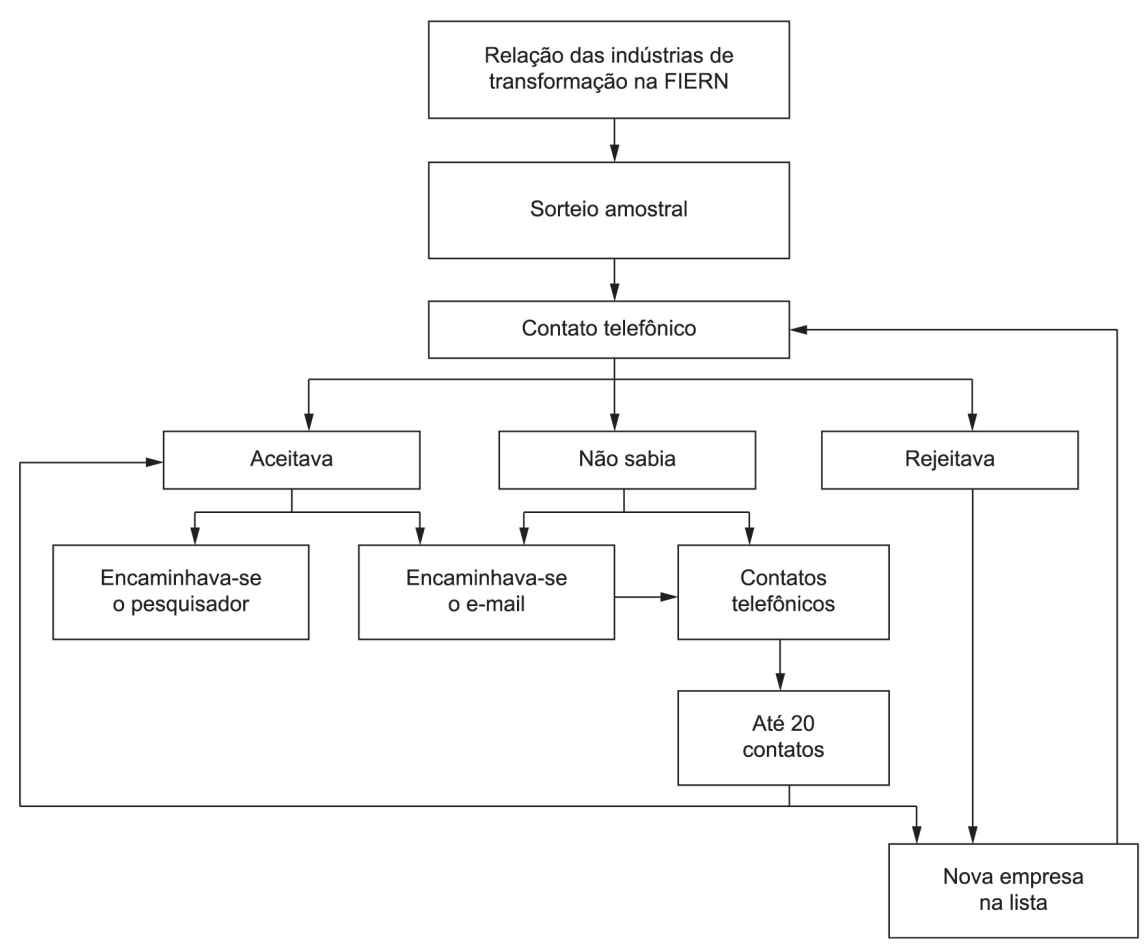

Figura 1. Fases da coleta dos dados. 


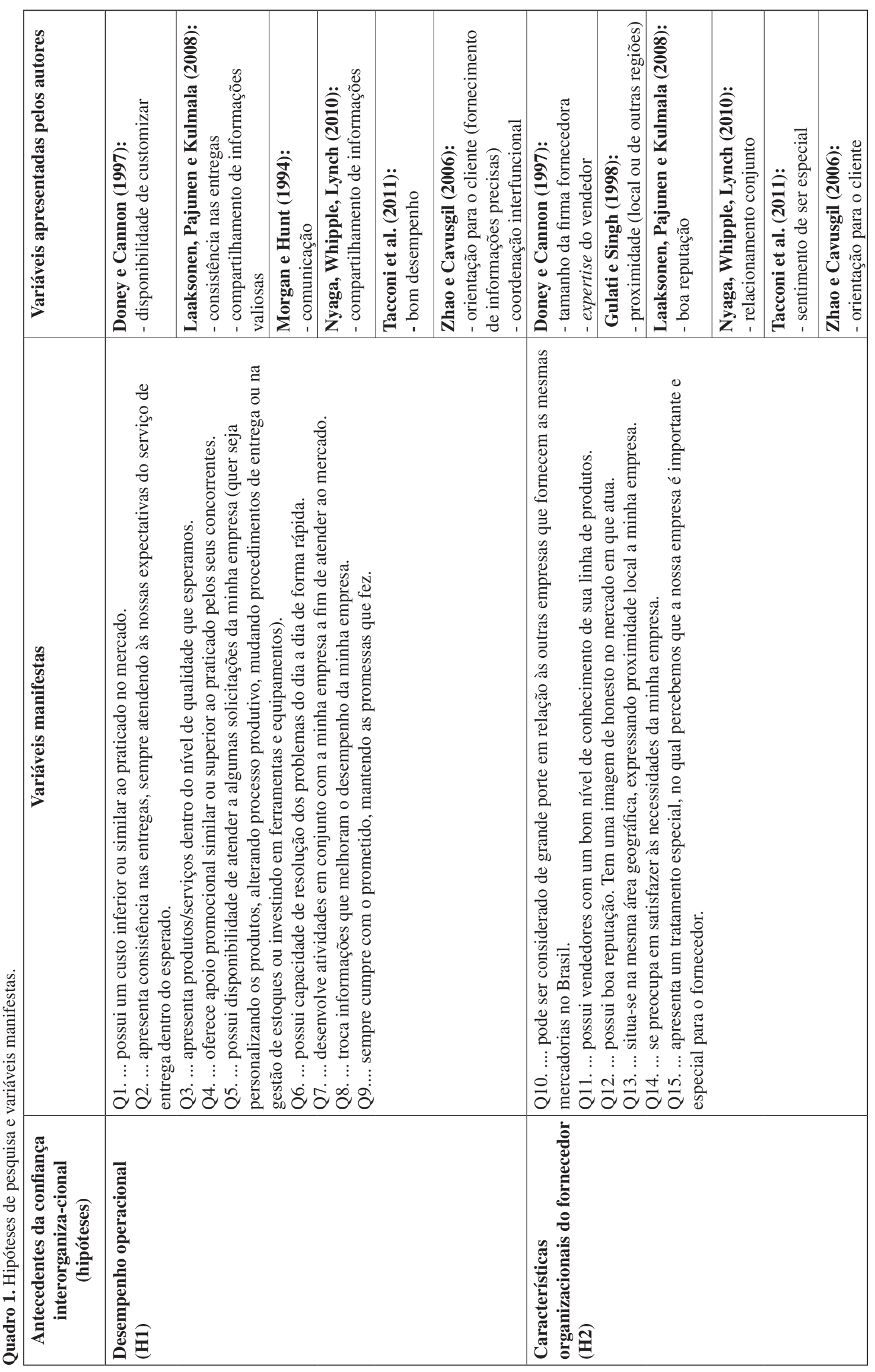




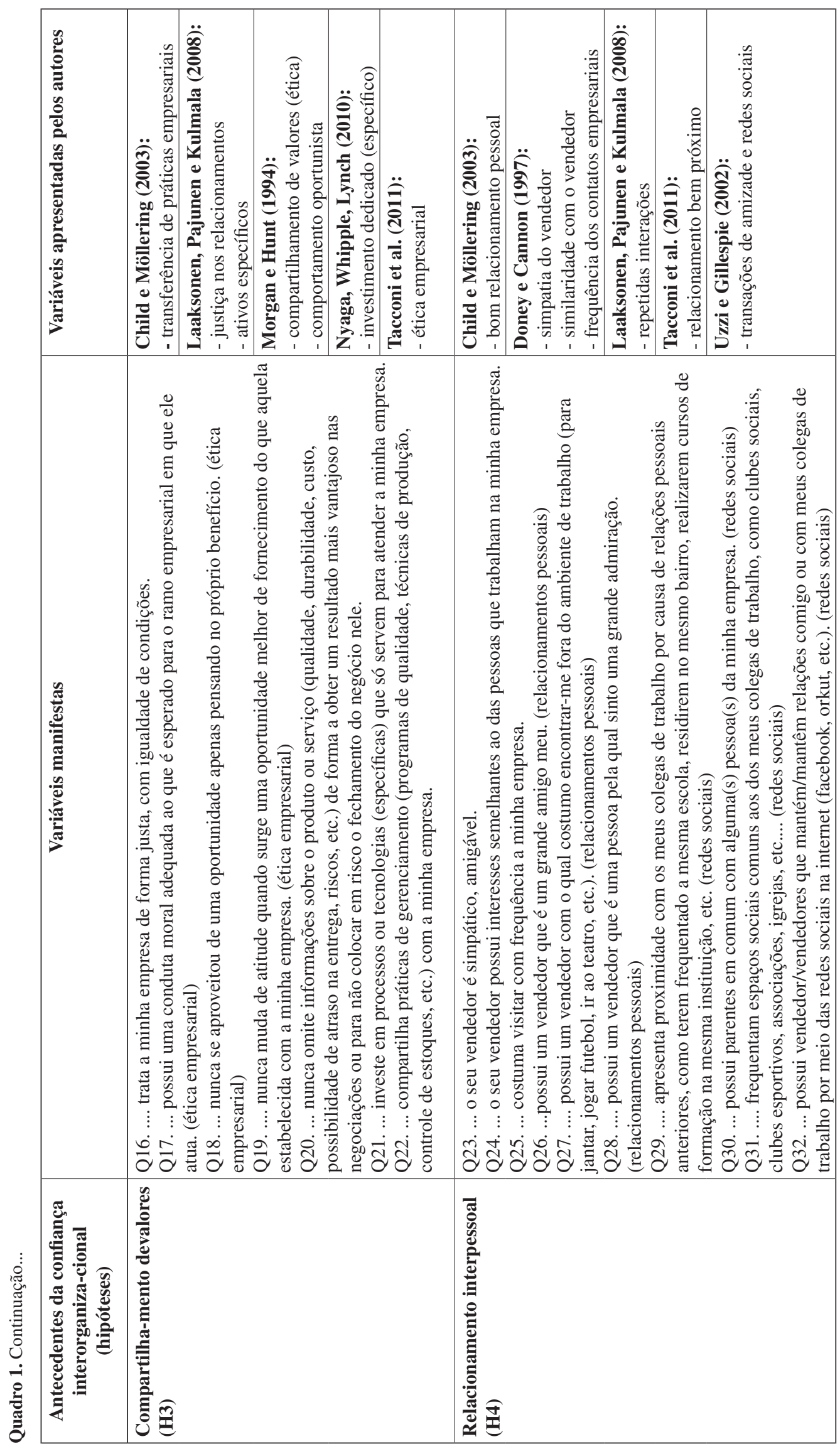


Para a modelagem de equações estruturais, inicialmente faz-se necessário verificar se houve alguma violação dos pressupostos para o bom desenvolvimento das análises. Nesse sentido, não foram identificados erros de codificação dos dados, não houve questões com escala invertida que necessitassem de recodificação e as não respostas, nesta pesquisa, foram esporádicas e analisadas como sem um padrão entre as variáveis que compõem os construtos que foram testados nas análises multivariadas. Por isso a correção escolhida para o banco de dados foi a substituição do valor da moda nos dados perdidos, o que possibilitou utilizar os 237 questionários na análise fatorial exploratória.

Em relação à confiabilidade das escalas utilizadas nos dados, foi realizado o teste Alfa de Cronbach. Verificando-se os resultados desse teste, assume-se que a escala de mensuração utilizada no instrumento de pesquisa apresenta boa aderência, refletindo consistência nos construtos e sendo satisfatória para a aplicação na análise multivariada.

Para a verificação da normalidade univariada, foram utilizados os testes Kolmogov-Smirnov e Shapiro-Wilk, que permitiram verificar que a hipótese nula $\mathrm{H}_{0}$ de normalidade foi rejeitada para cada uma das 32 variáveis a serem utilizadas na modelagem. Apesar de o teste Kolmogov-Smirnov ser útil para verificar a normalidade univariada, Marôco (2010) afirma que, de forma alternativa, costuma-se utilizar os testes de assimetria univariada - sk e curtose - ku para verificar a possibilidade de admissão da distribuição normal das variáveis.

Dada a presença de outliers (valores extremos) quando da verificação da normalidade multivariada das variáveis manifestas, foram identificados sete outliers mais extremos, isto é, com maiores distâncias de Mahalanobis $\left(D^{2}\right)$ e retirados os sete registros correspondentes no banco de dados original, que contava com 237 respondentes. Dessa forma, o banco de dados utilizado na análise da SEM apresenta 230 registros, minimizando a violação dos pressupostos da normalidade multivariada.

No entanto, nesta pesquisa, apesar da eliminação de sete outliers, a não normalidade univariada e multivariada dos dados foi verificada. Diante disso, optou-se por utilizar o método de reamostragens bootstrap na condução da modelagem de equações estruturais. As restrições para o uso do bootstrap são: que a amostra "original" seja considerada representativa da população; a distribuição das observações tenha a mesma escala, em virtude do uso de uma estrutura de covariância; e os dados não apresentem normalidade multivariada, porque o uso dessa ferramenta de reamostragem, em dados normais, leva a estimativas menos precisas do que as estimativas oriundas do método ML padrão. Mas, quando a distribuição é não normal, as estimativas bootstrap são melhores do que os resultados das estimativas de ML padrão (BYRNE, 2010). Por isso, seguindo-se essa compreensão de que os dados desta pesquisa não apresentam normalidade multivariada, que a amostra é representativa da população em estudo e que as observações foram todas medidas em uma mesma escala, optou-se pelo uso do método bootstrap com 500 reamostragens como a alternativa estratégica para reparar o problema da não normalidade multivariada encontrada nos dados, associado ao método da máxima verossimilhança - ML.

Para a análise de dimensionalidade dos dados da pesquisa, foi utilizada uma análise fatorial exploratória para cada uma das variáveis latentes. Essa análise permite verificar quais variáveis observáveis atuam juntas e quantas podem realmente ser consideradas como tendo impacto na análise a fim de apoiar a análise fatorial confirmatória. Na sequência, os resultados da análise fatorial exploratória foram transferidos para uma modelagem estrutural realizada em cada uma das quatro variáveis latentes (desempenho operacional, características organizacionais, compartilhamento de valores e relacionamento interpessoal).

\section{Buscando a explicação da confiança interorganizacional nas compras}

A partir da aplicação do instrumento, a amostra deste estudo foi conduzida em três momentos. Primeiro, são apresentados e analisados os aspectos do perfil sociodemográfico dos compradores e de suas organizações, localizadas na Grande Natal. No momento seguinte, verifica-se o nível de confiança interorganizacional apresentado pelos pesquisados, o tipo de produto adquirido e o tempo médio desse relacionamento pautado em confiança. $\mathrm{O}$ terceiro momento apresenta o processo de modelagem, apoiado por uma análise fatorial exploratória individual.

\subsection{Aspectos do perfil sociodemográfico do comprador e da organização compradora}

A proposta deste tópico é traçar o perfil do comprador das indústrias de transformação da cidade de Natal e de seu entorno, considerando-se algumas características, como gênero, idade, nível de educação, tempo de experiência no setor de compras e cargo que ocupa, assim como o perfil da organização compradora em relação ao ramo em que atua e ao porte, com base no número de funcionários.

Em relação ao gênero, é possível afirmar que quase $70 \%$ dos pesquisados são do sexo masculino, revelando que, nessa área, atuam preponderantemente homens. Quanto à faixa etária, os profissionais que efetuam as compras nas indústrias de transformação da Grande Natal estão bem distribuídos, havendo 
baixa incidência dos mais jovens, com até 20 anos, e de idosos, com mais de 60 anos.

No que se refere ao indicador escolaridade, quase $50 \%$ dos responsáveis pela atividade de compras e suprimentos têm o ensino médio completo, seguidos de $34 \%$ com ensino superior completo. Com menor incidência, encontram-se os compradores com pós-graduação completa (7\%), os que têm apenas o ensino básico (4\%) e os que não possuem sequer o ensino básico completo (quase 6\%).

Uma questão pertinente é o tempo de experiência profissional dos pesquisados na atividade de compras e suprimentos. Foi evidenciado que muitos entrevistados têm uma larga experiência na aquisição de suprimentos organizacionais: $53 \%$ estão há dez anos ou mais na atividade. Ao mesmo tempo, o percentual de novos profissionais nessa atividade também é representativo: aproximadamente $20 \%$ têm pouco tempo de atuação, até três anos.

Os sócios e proprietários foram os indivíduos com maior frequência na participação da pesquisa. Uma alternativa com grande número de participantes foi a opção "outros", formada em sua maioria, por diretores e gerentes administrativos, diretores e gerentes financeiros, gerentes comerciais, gerentes de logística e, em menor frequência, por supervisores, coordenadores de almoxarifado e filhos do proprietário. Essa alternativa (outros) apresentou esse resultado em virtude da diversidade de pessoas que assumem a função compras em uma organização. Segundo Baily et al. (2000), dependendo da importância atribuída à função compras, ela pode ser posta sob a responsabilidade de um gerente com interesses relacionados ou de um gerente exclusivo.

Todos os ramos de atividade das indústrias de transformação foram pesquisados, com maior predominância entre as indústrias de produtos alimentícios. Foram contemplados todos os portes organizacionais: $58,3 \%$ dos pesquisados trabalham em organização de porte micro (até 19 funcionários); $29 \%$ em organização de pequeno porte (entre 20 e 99 funcionários); $8 \%$ atuam em empresas de médio porte (entre 100 e 499 funcionários); e 4,7\% exercem suas funções em organizações de grande porte (500 ou mais funcionários).

\subsection{Nível de confiança interorganizacional, tipo de produto e tempo de relacionamento}

Como forma de enriquecer o estudo, foi verificado o nível de confiança interorganizacional, o tipo de produto adquirido e o tempo médio do relacionamento de confiança entre o fornecedor, ou conjunto de fornecedores, e a empresa compradora.

O resultado obtido foi que $3,4 \%$ apresentam um nível baixo de confiança (até 3,5); 14,8\% um nível intermediário de confiança (entre 4 e 5,5); e $72 \%$ um alto nível de confiança (igual ou superior a 7,5), significando que as indústrias de transformação da Grande Natal acreditam em um fornecedor, ou conjunto de fornecedores, de confiança, sentem-se seguros em relação a esse fornecedor, o que indica a existência desse fenômeno entre os pesquisados. Child (2001), Handfield e Bechtel (2002) e Laaksonen, Pajunen e Kulmala (2008) destacam, em seus trabalhos, a questão dos diferentes níveis de confiança que podem existir em um relacionamento.

A utilização do material no processo produtivo é um quesito que tende a diferenciar a forma de se processarem as atividades rotineiras de compras e suprimentos. Nessa direção, optou-se por analisar o tipo de produto que é adquirido do fornecedor, ou conjunto de fornecedores, de confiança. Os resultados demonstraram que $51 \%$ dos fornecedores de confiança suprem a empresa compradora com produtos que são utilizados diretamente no processo produtivo, seguidos por $44 \%$ que fornecem tanto produtos diretos quanto indiretos, e apenas 5\% das empresas compradoras depositam confiança em fornecedores de produtos utilizados indiretamente no processo produtivo.

Uma questão igualmente importante diz respeito ao tempo médio de relacionamento interorganizacional: em aproximadamente $44 \%$ dos casos estudados, esse tempo fica entre sete e doze anos de envolvimento com o fornecedor de confiança. Esse espaço de tempo já possibilita à empresa compradora conhecer realmente a forma de operação do fornecedor em diversas situações de aquisição. O levantamento também revelou que os relacionamentos com mais de vinte anos representam $8 \%$ das relações entre empresas compradoras e fornecedoras. Entretanto, é importante que as organizações ampliem seus relacionamentos para períodos de longo prazo, uma vez que isso possibilita maior troca de conhecimentos, adicionando valor às transações e contribuindo para o alcance dos objetivos estratégicos.

A essa análise descritiva, segue-se a modelagem de equações estruturais, que foi apoiada por uma análise fatorial exploratória.

\subsection{Equações estruturais: confirmando as hipóteses}

Em relação à dimensionalidade dos dados desta pesquisa foi utilizada uma análise fatorial exploratória para cada um dos construtos latentes. Essa técnica permite verificar quais variáveis observáveis atuam juntas e quantas podem realmente ser consideradas como tendo impacto nas relações, a fim de apoiar a análise fatorial confirmatória. Na sequência, os resultados da análise fatorial exploratória foram transferidos para uma modelagem estrutural, realizada em cada um dos quatro construtos (desempenho 
operacional, características organizacionais, compartilhamento de valores e relacionamento interpessoal). Os resultados dessas modelagens individuais foram agrupados em um modelo especificado dentro de um diagrama de caminhos, chamado de modelo inicial especificado. Para a estimação desse modelo, foi utilizado o método da máxima verossimilhança, por intermédio de quinhentas reamostragens (bootstrap), no software IBM SPSS Amos 18.0.

Verificando-se a configuração do modelo especificado e analisando-se os índices de ajustamento alcançados, a primeira tentativa de modelagem com todos os construtos não apresentou ajustamento. Segundo Marôco (2010), quando os testes não apresentam ajustes no modelo especificado, pode-se recorrer à reespecificação do modelo, em que se eliminam, de forma reduzida, as vias não significativas, fixando-se parâmetros que estavam livres, libertando-se parâmetros fixados anteriormente e/ou correlacionando-se erros de medida. Por isso, em virtude da falta de ajustamento do modelo inicial especificado, procedeu-se à reespecificação do modelo proposto. Com o modelo reespecificado, foi encontrada determinação do modelo, com níveis de ajustamento adequado.
Esse modelo reespecificado foi analisado com base em uma combinação de medidas de ajustamento que inclui medidas absolutas, comparativas e de parcimônia, para analisar a qualidade do ajustamento da modelagem. Essa combinação pode ser visualizada na Tabela 1, que apresenta as principais medidas de ajustamento do modelo reespecificado da confiança interorganizacional nas compras, comparado ao modelo inicial especificado para o estudo.

Os resultados da Tabela 1 confirmam que esse modelo reespecificado apresenta ajuste em relação ao RMSEA, ao GFI, ao $\chi^{2}$ g.g.l., ao CFI, ao NFI e ao TLI, bem como melhora os índices de ajustamento parcimonioso.

A última etapa, antes da decisão de aceitar ou rejeitar o modelo, é a validação deste, na qual se calculam a confiabilidade composta e a variância extraída, assim como se avaliam a validade convergente e a validade discriminante do modelo reespecificado. Esses cálculos e análises podem ser verificados nas Tabelas 2 e 3.

A Tabela 2 mostra que a confiabilidade composta das três variáveis exógenas apresentou consistência interna nos itens, revelando que existe relacionamento entre as variáveis observáveis de cada construto. E a variância extraída de cada construto excedeu o valor de 0,50 , significando que mais de $50 \%$ da variância

Tabela 1. Medidas do ajustamento do modelo reespecificado da confiança interorganizacional nas compras, comparado ao modelo inicial especificado.

\begin{tabular}{|c|c|c|c|}
\hline \multirow[b]{2}{*}{ Índices } & \multicolumn{2}{|c|}{ Resultados } & \multirow{2}{*}{$\begin{array}{c}\text { Nível de } \\
\text { aceitação } \\
\text { (KLINE, 1998) }\end{array}$} \\
\hline & $\begin{array}{c}\text { Modelo } \\
\text { inicial }\end{array}$ & $\begin{array}{c}\text { Modelo } \\
\text { reespecificado }\end{array}$ & \\
\hline Quiquadrado & 417,88 & 126,87 & \\
\hline Graus de liberdade (g.l.) & 118 & 84 & maior que 1 \\
\hline Nível de probabilidade & 0,000 & 0,002 & acima de 0,05 \\
\hline \multicolumn{4}{|l|}{ Medidas absolutas de ajustamento } \\
\hline Raiz do erro quadrático médio aproximado (RMSEA) & 0,105 & 0,047 & inferior a 0,08 \\
\hline Índice da bondade do ajustamento (GFI) & 0,821 & $\mathbf{0 , 9 3 0}$ & acima de 0,90 \\
\hline Quiquadrado normalizado ( $\left.\chi^{2} / \mathrm{g} .1.\right)$ & 3,541 & 1,510 & entre 0 e 3 \\
\hline \multicolumn{4}{|l|}{ Medidas comparativas de ajustamento } \\
\hline Índice de ajustamento comparativo (CFI) & 0,785 & 0,964 & acima de 0,90 \\
\hline Índice de ajustamento normalizado (NFI) & 0,727 & 0,901 & acima de 0,90 \\
\hline Índice de ajustamento Tucker-Lewis (TLI) & 0,753 & 0,954 & acima de 0,90 \\
\hline \multicolumn{4}{|l|}{ Medidas de parcimônia de ajustamento } \\
\hline Índice de parcimônia normalizado (PNFI) & 0,631 & 0,721 & acima de 0,60 \\
\hline Índice de parcimônia comparativo (PCFI) & 0,681 & 0,771 & acima de 0,60 \\
\hline
\end{tabular}

Tabela 2. Modelo de mensuração do modelo reespecificado.

\begin{tabular}{lcc}
\hline \multicolumn{1}{c}{ Construtos } & Confiabilidade composta (1) & Variância extraída (2) \\
\hline Desempenho operacional & 0,904 & 0,581 \\
Compartilhamento de valores & 0,814 & 0,593 \\
Relacionamento interpessoal & 0,875 & 0,586 \\
\hline
\end{tabular}


Tabela 3. Matriz de variâncias extraída e compartilhada dos construtos.

\begin{tabular}{lccc}
\hline \multicolumn{1}{c}{ Variâncias } & Desp_Oper & Comp_Valor & Relac_Interp \\
\hline Desempenho operacional & $\mathbf{0 , 5 8 1 ^ { \mathrm { a } }}$ & & \\
Compartilhamento de valores & $0,536^{*}$ & $\mathbf{0 , 5 9 3}^{\mathrm{a}}$ & \\
Relacionamento interpessoal & $0,074^{*}$ & $0,048^{*}$ & $\mathbf{0 , 5 8 6}^{\mathrm{a}}$ \\
\hline
\end{tabular}

avalores na diagonal são as variâncias extraídas; *Variância compartilhada entre as variáveis latentes.

dos indicadores é explicada pelo construto em questão. Para Hair Junior et al. (2005), a confiabilidade composta é considerada aceitável quando assume valores superiores a 0,70 , e a variância extraída é aceitável quando assume valores acima de 0,50.

A validade convergente também foi verificada, por meio do exame das cargas fatoriais dos indicadores na variável latente. Dessa forma, constata-se, no modelo reespecificado, a validade convergente para o modelo de mensuração dos construtos latentes desempenho operacional, compartilhamento de valores e relacionamento interpessoal, nos quais todas as variáveis indicadoras ou manifestas apresentam cargas fatoriais significativas (valor de C.R $=\mathrm{t}>2,58$ ) $\mathrm{e}$ correlações fortes $(>0,50)$. Portanto, todas as variáveis estão forte e significativamente relacionadas a seus construtos.

Outro ponto importante é a validade discriminante, verificável na Tabela 3.

Observando-se a Tabela 3 , percebe-se que a validade discriminante se configura para os três construtos do modelo reespecificado: desempenho operacional, compartilhamento de valores e relacionamento interpessoal, porque a variância extraída $\left({ }^{a}\right)$ de cada construto é superior à variância compartilhada (*).

A combinação dos resultados encontrados nos testes e análises da modelagem permite definir que o modelo reespecificado é fidedigno às variâncias da amostra estudada. Nessa direção, foram confirmadas as hipóteses H1, desempenho operacional; H3, compartilhamento de valores; e $\mathrm{H} 4$, relacionamento interpessoal, com efeito direto e positivo sobre a confiança interorganizacional nas compras; enquanto $\mathrm{H} 2$, característica organizacional, apresenta efeito indireto sobre esse fenômeno, conforme Figura 2.

O modelo reespecificado ajustado explica 99,7\% da variabilidade das respostas relativas à confiança interorganizacional nas compras, observada nas 230 respostas analisadas e que, na Figura 2, está apresentada de forma aproximada com o valor 1,00. Todas as trajetórias expostas nessa figura são positivas e significativas, assim como todos os pesos fatoriais dos itens de medida foram elevados. Esses resultados suportam a explicação de que a confiança interorganizacional nas compras depende diretamente do relacionamento interpessoal, do compartilhamento de valores e do desempenho operacional e, indiretamente, das relações pessoais, das redes sociais, das características organizacionais, dos aspectos físico e relacional do desempenho. Assim, essa confiança é explicada por um conjunto de interações entre esses três determinantes, nos quais o ponto central é o relacionamento interpessoal, como maior coeficiente de trajetória $(0,58)$.

Nesse sentido, o destaque desse modelo consiste na confirmação estatística da relação entre relacionamento interpessoal e confiança interorganizacional nas compras, além de o efeito de relacionamento interpessoal sobre confiança interorganizacional ser o mais pertinente nessas relações. Esses resultados corroboram as discussões de autores como Granovetter (1985), Uzzi e Gillespie (2002), Child e Möllering (2003), Perrone, Zaheer e McEvily (2003) e Tacconi et al. (2011).

Em relação à Figura 2, é possível observar que a variável exógena relacionamento interpessoal é formada por outras duas variáveis endógenas não observáveis, que são as redes sociais e o relacionamento pessoal. O relacionamento pessoal é formado por duas variáveis endógenas observáveis, que representam a amizade entre os atores organizacionais da empresa compradora e da fornecedora (Q26) e o vendedor, que é considerado uma pessoa pela qual o comprador tem uma grande admiração, estima (Q28).

Como o efeito de relacionamento pessoal e de redes sociais são positivos na modelagem, pode-se afirmar que, quanto maior o relacionamento pessoal entre comprador e fornecedor, assim como quanto maiores as redes sociais nas quais esses indivíduos estão imersos, maior será o efeito do relacionamento interpessoal sobre a confiança interorganizacional nas compras.

Em relação à modelagem, um ponto que também mereceu destaque foi o efeito de compartilhamento de valores sobre o modelo reespecificado. Essa variável exógena foi formada por três indicadores observáveis. $\mathrm{O}$ indicador $\mathrm{Q} 18$, relativo à ética, representa que o fornecedor de confiança ao qual o comprador se refere nunca se aproveitou de uma oportunidade pensando apenas no próprio benefício. Os outros indicadores referentes à ética mostram que o fornecedor de confiança nunca muda de atitude quando surge uma oportunidade melhor de fornecimento do que aquela estabelecida anteriormente (Q19) e que nunca omite informações a fim de obter vantagem nas negociações (Q20).

Das quatro variáveis latentes do modelo inicial especificado, a única que não apresentou efeito direto 


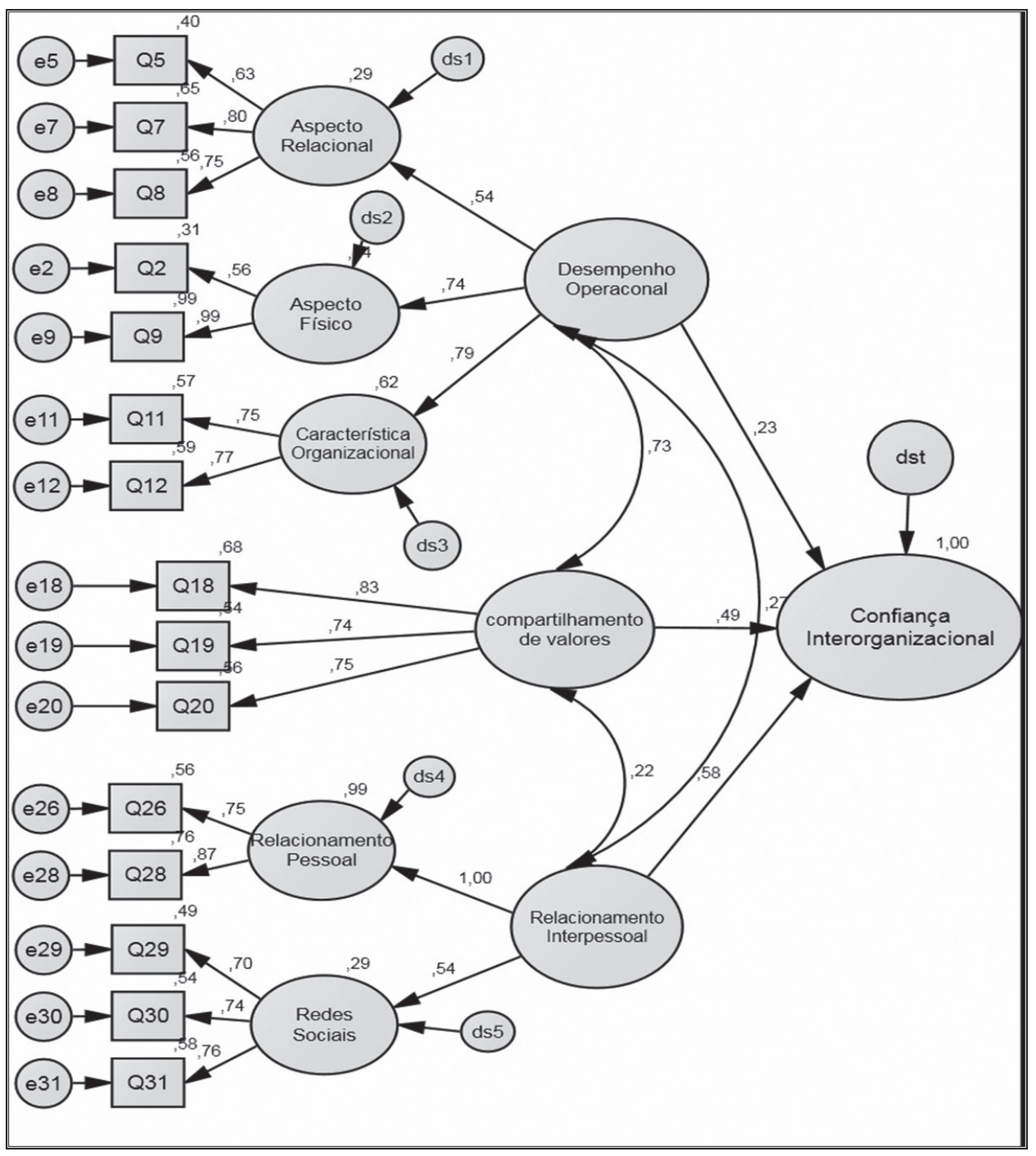

Figura 2. Estimativas padronizadas do modelo reespecificado da confiança interorganizacional nas compras.

significativo sobre a confiança interorganizacional foi características organizacionais ( $\mathrm{H} 2)$, mas essa variável apresentou efeito indireto por meio do construto desempenho operacional.

Verificando-se a Figura 2, a variável exógena desempenho operacional foi formada por três construtos latentes, denominados de características organizacionais, aspecto físico e aspecto relacional do desempenho operacional, que, por sua vez, são formados por sete indicadores.

O desempenho operacional foi o de menor efeito $(0,23)$. Essa informação pode ser um reflexo da percepção positiva que a organização compradora tem do fornecedor, porque, se este já tem algum tipo de amizade com o comprador, possui algum tipo de imbricamento social e apresenta uma imagem de ético no mercado em que atua, não tenderá a frustrar as expectativas de bom desempenho em relação a questões mais simples e objetivas, como entrega, troca de informações e outras.

Esses resultados contribuem para o desenvolvimento de relacionamentos diádicos e em redes, quer seja em áreas específicas da logística, como compras e suprimentos, quer seja na gestão das cadeias de abastecimento, especialmente pela confirmação das relações interpessoais como fator determinante na geração de confiança interorganizacional.

A análise da modelagem da confiança interorganizacional nas compras permitiu verificar que o modelo inicial proposto com os quatro construtos agrupados não apresentava ajustamento, precisando ser reespecificado. Já o modelo reespecificado apresenta um bom ajustamento à estrutura de variância e covariância dos itens analisados, nos quais todos os pesos fatoriais foram elevados, e explica bem a percentagem da variância das respostas sobre a confiança interorganizacional nas compras. 


\section{Conclusões}

Os compradores e demais membros organizacionais têm confiança interorganizacional, mas em diferentes níveis, corroborando as discussões sobre níveis de confiança de Child (2001), Handfield e Bechtel (2002) e Laaksonen, Pajunen e Kulmala (2008). No entanto, essa confiança interorganizacional tende a ser depositada nos fornecedores que suprem a empresa com os materiais que são utilizados diretamente no processo produtivo.

A modelagem permitiu concluir que o modelo de confiança interorganizacional nas compras formado pelo modelo reespecificado, com quinze variáveis endógenas observáveis, é satisfatório para responder a esse fenômeno. Isso confirma as hipóteses de que relacionamento interpessoal $(\mathrm{H} 4)$, compartilhamento de valores (H3) e desempenho operacional (H1) influenciam positivamente como antecedentes dessa confiança. No entanto, o relacionamento interpessoal foi a variável de maior impacto para a geração dessa confiança, o que vai ao encontro do que Granovetter (1985) defendia: a ação econômica está imersa nas redes sociais, que são influenciadas pelas relações pessoais. Assim, todos estariam emaranhados em relações pessoais, que estão imersas de forma mais ampla, nas redes sociais.

Considerando-se que a amostra é formada por $58 \%$ de microempresas e $29 \%$ de pequenas empresas, é possível inferir que as relações interpessoais assumem maior relevância. Tal destaque explica-se, entre outros aspectos, pelo fato de que quebra de contrato entre pequenas empresas tende a ter julgamento mais lento e a consumir significativos recursos dessas empresas - assim, a confiança funciona como mecanismo de coordenação interfirmas mais eficaz e mais econômico, e essa confiança se constrói principalmente a partir do fortalecimento das relações interpessoais. Depois, por a amostra ser de uma região formada por empresas que atuam predominantemente no mercado local, o fortalecimento das relações interpessoais é veículo fundamental para se obter reputação, o que, por sua vez, influencia positivamente no acesso a oportunidades de negócio. Esse segundo aspecto, por sua vez, aplica-se também para o caso de médias e grandes empresas, nas quais os relacionamentos interpessoais, como base da construção de relações de confiança, podem fortalecer e garantir a reputação positiva e, assim, oportunidades de novos negócios e solução rápida de problemas.

Nessa direção, sugere-se às organizações fornecedoras que desenvolvam formas de criar, fortalecer e ampliar as redes de relacionamentos pessoais e sociais existentes entre seus colaboradores e seus clientes, como forma de criar diferenciais competitivos difíceis de serem copiados. Além disso, a percepção do mercado industrial sobre os comportamentos antiéticos e oportunistas do fornecedor que pensa apenas no próprio benefício também se apresentou como uma questão pertinente para a geração de confiança.

Cientes da importância de desenvolver relacionamentos pessoais e sociais, assim como de refletir uma imagem ética no mercado em que atua, cabe às empresas preocuparem-se mais com os colaboradores que contratam para intermediar suas relações comerciais, bem como estarem atentas aos códigos morais, formais ou não, que disseminam dentro de suas organizações e que vão refletir-se na forma de fazer negócios com as outras empresas.

Esta pesquisa foi desenvolvida de forma criteriosa, mas tem suas limitações. Uma delas é que, como se refere à exploração de novas ideias, as relações interpessoais como antecedentes da confiança interorganizacional nas compras, requer repetições em futuros estudos.

O estudo foi desenvolvido na área de compras e suprimentos industriais e seria pertinente a reprodução em outras áreas organizacionais e em outros segmentos empresariais. Além disso, o foco recai na díade comprador-fornecedor como unidade de análise e assume a perspectiva da empresa compradora, mas, como esses relacionamentos envolvem os dois lados, seria importante examinar a natureza da relação de troca pela vertente da empresa fornecedora.

\section{Referências}

ARKADER, R. Compras e gerência de fornecimento no Brasil: estudos e casos. In: ARKADER, R. (Org.). Prefácio. Rio de Janeiro: Mauad, 2004. PMid:15073705.

BAIER, C.; HARTMANN, E.; MOSER, R. Strategic alignment and purchasing efficacy: an exploratory analysis of their impact on financial performance. Journal of Supply Chain Management, v. 44, n. 4, p. 36-52, Oct 2008. http://dx.doi. org/10.1111/j.1745-493X.2008.00071.x

BAILY, P. et al. Compras: princípios e administração. São Paulo: Atlas, 2000. PMid:10925466.

BARNEY, J. Firm Resources and Sustained Competitive Advantage. Journal of Management, v. 17 , n. 1, p. 99-120, 1991. http://dx.doi. org/10.1177/014920639101700108

BOWERSOX, D. J.; CLOSS, D. J.; COOPER, M. B. Gestão da Cadeia de Suprimentos e Logística. Rio de Janeiro: Elsevier, 2007. PMid:17389141.

BOWERSOX, D. J.; CLOSS, D. J. Logística Empresarial processo de integração da cadeia. São Paulo: Atlas, 2008.

BYRNE, B. M. Structural Equation Modeling with AMOS: basic concepts, applications, and programming. 2nd ed. New York: Taylor \& Francis Group, 2010. (Multivariate Applications Series).

CHEN, I. J.; PAULRAJ, A.; LADO, A. A. Strategic purchasing, supply management, and firm performance. Journal of Operations Management v. 22, p. 505-523, 2004. http://dx.doi.org/10.1016/j. jom.2004.06.002 
CHILD, J. Trust - the fundamental bond in global collaboration. Organizational Dynamics, v. 29, n. 4, p. 274-288, 2001. http://dx.doi.org/10.1016/ S0090-2616(01)00033-X

CHILD, J.; MÖLLERING, G. Contextual confidence and active trust development in the chinese business environmental. Organization Science, v. 14, n. 1, p. 69-80, Jan/Feb 2003. http://dx.doi.org/10.1287/ orsc.14.1.69.12813

CORREA, H. L. Oportunidades de pesquisa e ensino na área de operações no cenário internacional. In: ENCONTRO NACIONAL DE PÓS-GRADUAÇÃO EM ADMINISTRAÇÃO, 34., 2010, Rio de Janeiro. Anais... Rio de Janeiro: EnANPAD, 2010.

DAS, T. K.; TENG, B.-S. The risk-based view of trust: a conceptual framework. Journal of Business and Psychology, v. 19, n. 1, p. 85-116, 2004. http://dx.doi. org/10.1023/B:JOBU.0000040274.23551.1b

DONEY, P. M.; CANNON, J. An Examination of the Nature of Trust in Buyer-Seller Relationships. Journal of Marketing, v. 61, n. 2, p. 35-51, 1997. http://dx.doi. org/10.2307/1251829

EUROPEAN GROUP FOR ORGANIZATIONAL STUDIES - EGOS. 27th EGOS Colloquium - Gothenburg - 2011 - Sub-theme 02: Organizational Trust: Challenges and Dilemmas. Disponível em: <http://www.egosnet.org/jart/prj3/ egosnet/main.jart>. Acesso em: 20 jan. 2011.

FEDERAÇÃO NACIONAL DAS INDUSTRIAIS DO ESTADO DO RIO GRANDE DO NORTE - FIERN. Cadastro Industrial. Disponível em: <http://www. fiern.org.br>. Acesso em: 11 jul. 2011.

FIGUEIREDO, K.; ARKADER, R. Da Distribuição Física ao Supply Chain Management: o Pensamento, o Ensino e as Necessidades de Capacitação em Logística. Tecnologística, v. 4, n. 33, ago. 1998.

GANESAN, S.; HESS, R. Dimensions and levels of trust: implications for commitment to a relationship. Markeing Letter, v. 8, n. 4, p. 439-448, 1997. http:// dx.doi.org/10.1023/A:1007955514781

GRANOVETTER, M. Economic action and social structure: The problem of embeddedness. American Journal of Sociology, v. 9, n. 3, p. 481-510, 1985. http://dx.doi. org/10.1086/228311

GULATI, R.; SINGH, H. The architecture of cooperation: managing coordination costs and appropriation concerns in strategic alliances. Administrative Science Quarterly, n. 43, p. 781-814, 1998. http://dx.doi. org/10.2307/2393616

HAIR JUNIOR, J. F. et al. Análise multivariada de dados. 5. ed. Porto Alegre: Bookman, 2005.

HANDFIELD, R. B.; BECHTEL, C. The role of trust and relationship structure in improving supply chain responsiveness. Industrial Marketing Management, v. 31, p. 367-382, 2002. http://dx.doi.org/10.1016/ S0019-8501(01)00169-9

HERNANDEZ, J. M. C.; SANTOS, C. C. Proposta para um modelo de mensuração de confiança entre canais de marketing. In: ENCONTRO NACIONAL DE PÓS-GRADUAÇÃO EM ADMINISTRAÇÃO, 31., 2007, Rio de Janeiro. Anais... Rio de Janeiro: EnANPAD, 2007.
HILL, J. A. et al. The effect of unethical behavior on trust in a buyer-supplier relationship: the mediating role of psychological contract violation. Journal of Operations Management, n. 27, p. 281-293, 2009. http://dx.doi. org/10.1016/j.jom.2008.10.002

KLINE, R. B. Software Review: Software Programs for Structural Equation Modeling: Amos, EQS, and LISREL. Journal of Psychoeducational Assessement, v. 16, n. 4, p. 343-364, 1998. http:// dx.doi.org/10.1177/073428299801600407

KNIGHT, L. A. Learning to collaborate: a study of individual and organizational learning, and interorganizational relationships. Journal of Strategic Marketing, v. 8, p. 121-138, 2000. http://dx.doi. org/10.1080/096525400346213

LAAKSONEN, T.; PAJUNEN, K.; KULMALA, H. I. Co-evolution of trust and dependence in customer-supplier relationships. Industrial Marketing Management, n. 37, p. 910-920, 2008. http://dx.doi.org/10.1016/j. indmarman.2007.06.007

LEWICKI, R. J.; TOMLINSON, E. C.; GILLESPIE, N. Models of interpersonal trust development: theoretical approaches, empirical evidence, and future directions. Journal of Management, n. 32, p. 991-1022, 2006. http://dx.doi.org/10.1177/0149206306294405

MARÔCO, J. Análise de Equações Estruturais: fundamentos teóricos, Software \& aplicações. ReportNumber. 2010.

MORGAN, R. M.; HUNT, S. D. The Commitmenttrust theory of relationship marketing. Journal of Marketing, v. 58, n. 3, p. 20-38, 1994. http://dx.doi. org/10.2307/1252308

MORRISSEY, W. J.; PITTAWAY, L. Buyer-Supplier relationships in small firms: the use of social factors to manage relationships. International Small Business Journal, v. 24, n. 3, p. 272-298, 2006. http://dx.doi. org/10.1177/0266242606063433

NYAGA, G. N.; WHIPPLE, J. M.; LYNCH, D. F. Examining supply chain relationships: Do buyer and supplier perspectives on collaborative relationships differ? Journal of Operations Management, v. 28, p. 101-114, 2010. http://dx.doi.org/10.1016/j.jom.2009.07.005

PERRONE, V.; ZAHEER, A; McEVILY, B. Free to be trusted? Organizational Constraints on trust in boundary spanners. Organization Science, v. 14, n. 4, p. 422-439, 2003. http://dx.doi.org/10.1287/ orsc. 14.4.422.17487

POWELL, W. W. Hybrid Organizational Arrangements: New Form or Transitional Development? California Management Review, v. 30, n. 1, p. 67-87, 1987. http:// dx.doi.org/10.2307/41165267

SEPPÄNEN, R.; BLOMQVIST, K.; SUNDQVIST, S. Measuring inter-organizational trust - a critical review of the empirical research in 1990-2003. Industrial Marketing Management, v. 36, p. 249-265, 2007. http://dx.doi.org/10.1016/j.indmarman.2005.09.003

TACCONI, M. F. F. S. et al. R. Explorando os Geradores da Confiança nas Compras Interorganizacionais. Revista Portuguesa e Brasileira de Gestão, v. 10, n. 3, p. 37-47, jul./set. 2011.

TACCONI, M. F. F. S.; LOPES, F. D.; RAMOS, A. S. M. A Confiança e as Relações de Compra: o Caso 
da Queiroz Galvão Alimentos. In: ENCONTRO DE ESTUDOS EM ESTRATÉGIA, 4., 2009, Recife. Anais... Pernambuco: 3E's, 2009.

UZZI, B.; GILLESPIE, J. J. Knowledge spillover in corporate financing networks: embeddedness and the firm's debt performance. Strategic Management Journal, v. 23, p. 595-618, 2002. http://dx.doi.org/10.1002/smj.241

VIANA, F. L. E.; BALDI, M. Contribuições da Teoria de Redes à Gestão da Cadeia de Suprimento. In: ENCONTRO NACIONAL DE PÓS-GRADUAÇÃO EM ADMINISTRAÇÃO, 32., 2008, Rio de Janeiro. Anais... Rio de Janeiro: EnANPAD, 2008.

WALTER, J.; LECHNER, C.; KELLERMANNS, F. W. Knowledge transfer between and within alliance partners: private versus collective benefits of social capital. Journal of Business Research, v. 60, p. 698-710, 2007. http:// dx.doi.org/10.1016/j.jbusres.2007.01.026

WILLIAMSON, O. E. Economics and organization: a primer. California Management Review, v. 38, n. 2, p. 131-146, 1996. http://dx.doi.org/10.2307/41165836

ZAHEER, A.; HARRIS, J. Interorganizational trust. In: SHENKAR, O.; REURER, J. J. (Coord.). Handbook of Strategic Alliances. Thousand Oaks: Sage, 2006. http://dx.doi.org/10.4135/9781452231075.n10

ZHAO, Y.; CAVUSGIL, S. T. The effect of supplier's market orientation on manufacturer's trust. Industrial Marketing Management, n. 35, p. 405-414, 2006. http://dx.doi.org/10.1016/j.indmarman.2005.04.001 